\title{
Future antibacterial strategies: from basic concepts to
}

\section{clinical challenges}

Vincent Cattoir ${ }^{1,2} \&$ Brice Felden ${ }^{1}$

1University of Rennes 1, Inserm, BRM [Bacterial Regulatory RNAs and Medicine] UMR_S 1230, F-35043 Rennes, France. ${ }^{2}$ Rennes University Hospital, Department of Clinical Microbiology \& National Reference Center for Antimicrobial Resistance ('Enterococci' lab), F-35033 Rennes, France.

*Correspondence: Prof. Vincent Cattoir, CHU de Rennes, Service de Bactériologie-Hygiène hospitalière, 2 rue Henri Le Guilloux, 35033 Rennes Cedex, France. +33-2-99-28-98-28, Fax: +33-2-99-28-41-59, E-mail: vincent.cattoir@chu-rennes.fr or to Prof. Brice Felden, Email: bfelden@univ-rennes1.fr.

Keywords: Antimicrobial resistance; Multidrug resistance; Antibacterials; Therapeutic alternatives. 


\begin{abstract}
Conventional antibiotics have cured more diseases than all other drug classes combined. However, the pipeline of novel antibiotics is running dry while multi-drug resistance (MDR) is increasing at an alarming rate. Even though vaccines and antibodies are part of the toolkit against antimicrobial resistance, broadening the concept of 'antibiotic' is essential for innovation. Original roadmaps are needed to tackle the overwhelming MDR bacterial infections. The majority of 'in-use' antibacterial drugs derived from secondary metabolites that are produced by cultured soil microorganisms. Ongoing investigations extend large scale microbiome analyses to new natural sources, target the host-pathogen interface, include nanotechnologies and rejuvenate old paths, such as phages and bacteriocins. Host immune modulators and virulence-resistance suppressors are also being developed, with RNA therapeutics. These strategies should help to improve future prevention and treatment of MDR bacterial infections, but numerous challenges limit their introduction into clinical practice.
\end{abstract}




\section{Introduction}

Bacterial resistance to antibiotics has dramatically increased and is a major threat for public health in the $21^{\text {st }}$ century [1]. By 2050, the total number of deaths resulting from antimicrobial resistance is projected to rise to 10 million per year worldwide [2]. Major multidrug-resistant (MDR) opportunistic microorganisms are spreading, particularly those belonging to the ESKAPE group, which comprises Enterococcus faecium, Staphylococcus aureus, Klebsiella pneumoniae, Acinetobacter baumannii, Pseudomonas aeruginosa and Enterobacter spp. [3]. The ongoing clinical pipeline includes approximately 50 new antibacterial drugs possessing activity against priority pathogens, but is confined to derivatives of established antibiotic classes (the so-called 'me-too' drugs) [4]. Since very few novel classes of antibacterial agents have been discovered, there is an urgent need to propose and develop alternative strategies against bacterial pathogens, especially MDR Gram-negative bacteria, Mycobacterium tuberculosis, and Clostridium difficile [5]. Several alternative strategies have been proposed usually based on efficacy, but it is not the only, and possibly not even the most important, constraint on the development of new antibacterial strategies. Indeed, the real challenge lies in clinical and practical aspects such as safety, 'drugability', approval process, and economics. This narrative review presents the main preventive and therapeutic approaches that have been developed to potentially guide drug discovery of novel antimicrobial agents (Figure 1).

\section{Naturally-produced drugs and agents with novel mechanisms of action}

During the golden age of antibiotic development, drugs were mostly discovered by screening microbial natural products from fermentation broths. Thus, natural products are 
a historically successful and still a viable option as sources for new antibiotics. The prime requirement for screening natural products is to identify novel chemical scaffolds sustaining antibacterial activity. Most existing antibiotic classes are from natural compounds, with only a few exceptions entirely conceived through synthetic chemistry. Empirical natural products screenings were traditionally successful since antibiotics conferred competitive advantages in hostile and crowded microbial environments. For instance, soil is a unique source of microbial diversity, with up to 5,000 species per gram of soil containing approximately $10^{9}$ bacteria. Conventional culture techniques lead to the recovery of only ca. $1 \%$ of the microbial soil population and the development of methods to isolate 'unculturable' members of complex microbial communities can be considered as an untapped source of new antibiotics [6]. Recently, this approach led to the discovery of a novel compound from soil, named teixobactin, which inhibits Gram-positive and mycobacterial cell wall synthesis by binding to peptidoglycan and teichoic acid precursors [7]. Also, a culture-independent natural product discovery platform has recently led to a new class of antibiotics extracted from dirt, produced by the soil microbiome [8]. Furthermore, research has been extended to other natural sources such as the oceans $(\sim 1 \mathrm{x}$ $10^{6}$ bacteria per $\mathrm{ml}$ of water in the coastal ocean), lakes with $\sim 2$ to $4 \times 10^{6}$ bacteria per $\mathrm{ml}$ of water in eutrophic lakes (an increase into the rate of supply of organic matter), rivers, sediments, urban soils, or from plants, marine invertebrates, insects and vertebrates [9]. Metagenomics is a powerful culture-independent technique that could allow accessing the collective genomes of bacterial populations in natural habitats, to discover new antibiotic producers [10]. Genomic analysis reveals that many microorganisms have far greater potential to produce specialized metabolites than is suggested by classic bioactivity 
screens. In environmental bacteria, most antibiotic biosynthetic gene clusters (BGCs) are not expressed under laboratory conditions and are termed 'silent' or 'cryptic'. Thus, naturally-derived drugs were acquired from a minor fraction of constitutively expressed biosynthetic gene clusters, and methods that activate silent BGCs producing novel metabolites would significantly enhance drug discovery [9]. Growth-inhibitory molecules, including antibiotics, can induce the expression of silent BGCs, suggesting that old antibiotics may be used to find new ones [11]. However, despite allowing to access an unexplored chemical space, converting a natural product into an antibacterial usable in clinics remains a difficult task, for a number of reasons. Natural products are sometimes difficult to produce at scale, can lack drug-like properties in vivo, and are often difficult to modify chemically to optimize efficacy.

Clinically-used antibiotics mainly target bacterial nucleic acids, proteins and peptidoglycan syntheses. However, the design of structurally new antibacterial agents possessing novel mechanisms of action and new targets remains a promising strategy. For instance, several new inhibitors have been developed, such as non-fluoroquinolone bacterial topoisomerase inhibitors, cell division (FtsZ) inhibitors, peptide deformylase inhibitors, fatty acid synthesis (FabI) inhibitors, as well as agents affecting energy metabolism and bacterial membrane-active compounds [12].

\section{Drug modifications, hybrids and nanoparticles}

Structural alterations of existing antibiotic classes can increase potency and activity spectrum against pathogens, reverse or avoid emergence of resistance mechanisms, attenuate host toxicity and ecological impact on commensal microbiota, and/or improve 
drug pharmacokinetics. Such modification of existing antibiotics is a classic strategy that has been successful, one key example being the $\beta$-lactams [13]. Furthermore, fusing two or more pharmacophores belonging to an established agent known to elicit a desired antimicrobial effect is an old concept that has also been rejuvenated [14]. These antibiotic hybrids may be used as stand-alone antibacterial agents or as adjuvants that potentiate the activity of a primary antibiotic. An interesting example is the recent design of glycopeptide analogs that overcome the molecular basis of vancomycin resistance thanks to the introduction of two modifications, providing two additional independent and synergistic mechanisms of action not found in the parental antibiotic [15]. These vancomycin modifications also lower propensity for acquired resistance by vancomycin-resistant enterococci. Even though these vancomycin derivatives are far from approval, this proof of concept has already been validated since there are several hybrid drug candidates currently in clinical trials. For instance, cadazolid (consisting of fused pharmacophoric portions derived from ciprofloxacin and tedizolid) has completed phase 3 clinical trials for the treatment of $C$. difficile infection, and the cephalosporin-siderophore hybrid cefiderocol is currently in phase 3 clinical trials for the treatment of severe infections caused by carbapenem-resistant Gram-negative bacteria [4].

New materials, such as nanoparticules (NPs) that are between 0.1 and $100 \mathrm{~nm}$, can be designed to treat bacterial MDR infections. As an example to improve antibacterial delivery, an effective antibacterial was engineered using a peptide-based toxin (a peptide library was screened) enclosed in a biodegradable porous silicon NP delivery vehicle, active for treating P. aeruginosa lung infections [16]. Also, several types of inorganic and organic NPs, including various metals (especially gold and silver), were developed and evaluated in vitro 
[17]. Since the mechanisms of action of NPs are not well understood and this field of research is in its infancy, the potential risks of toxicity associated with using NPs in healthcare are not known yet [18].

\section{Phages and endolysins}

Phage therapy to treat bacterial infections with lytic bacteriophages, alone or in combination with traditional antibiotics, is a promising strategy to combat MDR infections [19]. They have been tested in eastern countries (e.g., Georgia, Poland) for treating infections due to bacteria resistant to conventional antibiotics [20]. Whereas phages are harmless to eukaryotic cells from patients, there are several obstacles to their use in clinics: they are difficult to standardize and there is a need to employ cocktails of multiple phages due to their narrow spectra; there are differences in biological, physical, and pharmacological properties of phages compared to conventional antimicrobials; there is a relative lack of regulatory approval processes. In addition, the emergence of bacterial resistance to phages could be a problem in chronic conditions (e.g. cystic fibrosis) that require long-term therapy [20]. Note that some phase $1 / 2$ clinical trials have been conducted or are in progress, but all investigate safety/efficacy of phage therapy as topical treatments [21].

Bacteriophage-derived enzymes, such as endolysins and other peptidoglycan hydrolases that disrupt bacterial cell walls, represent interesting alternatives to conventional antibiotics [22]. Indeed, endolysins are enzymes used by bacteriophages at the end of their replication cycle to degrade the peptidoglycan layer of their bacterial hosts triggering lysis. Molecular engineering of endolysins has been employed to develop novel antimicrobials 
[23]. These lytic enzymes exhibiting a high degree of host specificity could potentially replace or be utilized in combination with antibiotics. LysK and its derivatives are among the best-characterized endolysins, which show activity against multiple staphylococcal species [24]. Some candidates are currently under phase 2 clinical trials, but some of them can be responsible for allergic reactions due to their proteinaceous nature. Note that endolysins are ineffective against Gram-negative bacteria since the outer membrane prevents their access to the peptidoglycan.

\section{Vaccines and antibodies}

Attachment proteins from the surface of eukaryotic cells and pathogens are essential components for cell adhesion or signaling, and are primary targets for vaccine development and therapeutic antibodies. Widespread vaccination against bacterial pathogens is one of the first lines of defense against MDR infections since vaccines can prevent infection, with no need for antibiotic treatment [25]. Vaccines reduce the prevalence of resistance by lowering the need for antimicrobial use and by diminishing the total number of cases [26]. Development of new and effective vaccines, however, cannot proceed without a detailed understanding of the immune correlates of protection. Also, the development of vaccines against bacterial pathogens requires a deep understanding of how vaccination affects the growth and spread of the bacteria within the host. This provides essential information for vaccine design, and guides the parameters to be measured when evaluating vaccine efficacy. Vaccine development has often led to disappointing clinical trials results or even serious safety concerns [27]. 
The use of pathogen-specific monoclonal antibodies (mAbs) could prevent or treat an infection [28]. Preclinical data suggest that mAbs can also act synergistically with antibiotics. As targeted accurate therapies, they are less prone to induce broad resistance among bacteria, and should not disturb the healthy microbiome to any great extent. Surface antigens are popular targets for antibacterial antibody discovery. The key activities of bacterial surface-specific mAbs are engagement of the host immune system through complement fixation and opsonophagocytic killing (OPK). However, clinical trials with mAbs targeting $S$. aureus cell surface antigens lipoteichoic acid (pagibaxumab) and ClfA (aurexis) were unsuccessful, lacking OPK activity or the ability to bind bacteria in vivo. Antibodies against bacterial toxins might be smart options to prevent resistances because their effects should not feed back to the genome. mAbs can be designed with multiple mechanisms of action in a single molecule. Efficient receptor inhibition can be achieved by using an antibody that binds the binding site and displaces the ligand. As an example, monoclonal antibodies against Escherichia coli mannose-binding adhesin FimH inhibit and reverse bacterial adhesion [29]. In addition, bezlotoxumab (MK-6072), a monoclonal antibody against Clostridium difficile toxin B recently approved by the US Food and Drug administration (FDA), has emerged as a novel dynamic adjunctive therapy for prevention of recurrent $C$. difficile infections [30]. It is a safe and well-tolerated drug with low risk of serious adverse events or drug-drug interactions.

Finally, an interesting approach is to combine an antibody with an antibiotic. For instance, an antibody-antibiotic conjugate (AAC) was designed, consisting of an anti-S. aureus antibody covalently linked to an antibiotic. The resulting AAC has no antibacterial activity against planktonic $S$. aureus, but when AAC opsonized bacteria are taken up by host cells, 
intracellular proteases cleave the internal linker and release the active antibiotic [31]. Limitations of vaccines and antibody therapies are their potential negative impacts on commensal bacteria from the microbiota and their limited effectiveness due to the variability of the components at the bacterial surface. Also, the high cost of mAbs may also limit their potential use as alternative treatments, especially in low- and middle-income countries.

\section{Immune modulators and virulence suppressors}

Infections can be viewed as perturbations of host-pathogen interactions, inducing disequilibria between host immunity and bacterial growth. Host immunity is an important, but often overlooked factor in the clearance of drug-resistant infections. Our body harbors around 10 times more bacteria than human cells. Disturbances in this subtle and complex balance can displace host-microbe interactions from symbiosis to infection. Pathogens actively suppress host immune responses by expressing specific factors and regulators, in turn contributing to pathogenicity and disease onset. Host-directed immunomodulatory therapies are promising approaches wherein natural host mechanisms are exploited to enhance therapeutic benefits [32]. Strategies include (i) targeting innate immune receptors, (ii) use of innate defense regulator peptides and (iii) bacterial signaling molecules. Several immune modulators have been developed to boost the effectiveness of antimicrobial therapies by enhancing both innate and adaptive immune responses in the infected hosts (the so-called 'immune boosters'). Considering infection as a perturbation of the hostpathogen interactions, novel drugs seek to modulate host immune cells, strengthen phagocyte killing, and stimulate expression of antimicrobial effectors [33]. Host-defense 
peptides modulate the innate immune response and boost infection-resolving immunity while dampening potentially harmful pro-inflammatory responses (to reduce the 'off-target effects' of the host response), and were proposed as starting points to design novel antibiotics [34]. Despite nearly three decades of design efforts, however, there has been limited clinical success, due to potential toxicity, susceptibility to proteases, and high production costs [35]. Also, drug development efforts focusing on the regulation of innate defenses have been limited due to the potential for inducing harmful sepsis responses. Pharmaceutical exploitation of the innate immune response (TLR agonists as an example) was restricted to cases where stimulation of inflammation was contained or managed by localized or low dosing [36].

Virulence factor neutralization prevents pathogens from deploying their fighting devices during infection, while being more susceptible to clearance [37]. Targeting specific virulence factors, such as secretion systems and toxins, can lead to novel anti-infective therapies. Among others, protection can be achieved through quorum sensing (QS) inhibitors [38]. Also, anti-adhesion molecules can impede host cells attachment. A promising broad-spectrum target is the poly-N-acetylglucosamine (PNAG), which is a conserved surface polysaccharide produced by almost all bacterial pathogens. PNAG-based immunotherapies and vaccines for humans were successfully tested, such as mAb F598 in a phase I clinical trial [39]. Secreted cytotoxins that damage host cells can also be trapped and inactivated, or their assembly prevented. Targeting bacterial virulence factors can halt disease progression and also enhance host immune clearance [33]. 


\section{Microbiota modifications}

Alterations to the host microbiota can have profound detrimental consequences on human health. The human gut microbiota contains about 100 trillion microbial cells and impacts the overall human physiology, especially metabolism, nutrient absorption and immune functions. The human microbiome is overly exposed to antibiotics due to their intensive medical use and utilization in animal farms and crops. Devising novel narrow-spectrum therapeutics that only target bacterial pathogens, while minimizing deleterious effects to the human microbiota, is highly desirable [40]. Gut microbiota can be manipulated through active strategies using probiotics, prebiotics, or synbiotics (synergistic combinations of probiotics and prebiotics) [9]. Most probiotics are from lactic acid bacteria, and their effects on the digestive flora depend on the bacterial strain and are determined by bacteriocin production [41]. Indeed, probiotics produce various antibiotics that can inhibit or kill other gut microbes, and they can also inhibit growth of pathogenic bacteria. Also, some bacterial metabolites can possess powerful antibacterial activities without negative effect on the human microbiota. Therapeutic strategies recently emerged with the advent of fecal microbiota transplant (FMT), and were successfully applied for treating C. difficile infections [42]. Note that alterations of the microbiota appear unlikely to offer a therapeutic option for acutely ill patients and some potential long-term risks could be related to FMT such as weight gain.

\section{Antibiotic resistance inhibitors}

There are many examples of antimicrobial resistance inhibitors already used in clinical practice or under development, such as $\beta$-lactamase and efflux pump inhibitors $[13,43]$. 
Since $\beta$-lactamase production represents the most relevant mechanism of resistance in MDR Gram-negative pathogens, there is a significant regain of interest towards new $\beta$ lactamase inhibitors (BLIs) [44]. Among them, three novel non- $\beta$-lactam compounds (i.e., avibactam, vaborbactam, and relebactam) are clinically available or in clinical trials [45]. Several efflux pump inhibitors (EPIs) have been designed but their development was stopped for most of them because their molecular scaffolds must be optimized for higher activity and less toxicity [46]. No EPI is used in clinics up to now.

Besides, there are also interesting approaches to prevent dissemination of antibiotic resistance genes among bacteria. An example is the use of specific conjugation inhibitors (COINs) to prevent or lower plasmid dissemination [47]. Another promising strategy is to limit selective pressure of antibiotic residuals excreted into the gut on the microbiota either by antibiotic degradation (e.g. $\beta$-lactam inactivation by ribaxamase, currently in phase 2 clinical trials) or by antibiotic sequestering through adsorption (e.g. DAV132, currently in phase 1 clinical trials), in turn minimizing the emergence of resistance [48,49].

\section{Antimicrobial peptides, bacteriocins and predatory bacteria}

Antimicrobial peptides (AMPs), also called host defense peptides, are part of the innate immune response found among all classes of life [50]. They are potent, broad-spectrum antibiotics also active against fungi and viruses, by acting via membrane permeabilization. Numerous products are in pre-clinical developments but several obstacles (such as proteolytic instability, short in vivo half-life, insolubility, poor bioavailability, and toxicity issues) hamper their translation into therapeutic drugs [9]. Only two AMPs (Lytixar and Pexiganan) are in clinical trials, only for topical use [46]. Amongst AMPs, defensins are 
cationic compounds that form pore-like structures with permeability enhancement. Noteworthy, a defensin-mimetic compound (brilacidin) is currently in phase 2 clinical trials for acute bacterial skin and skin-structure infections [46].

Bacteriocins are gene-encoded, ribosomally processed antimicrobial peptides produced by bacteria against other bacteria, while they are usually non-toxic to human cells. Bacteriocin incorporation into drug delivery systems, as alternatives to conventional antibiotics, is an emerging field. They can target cell walls (nisin) or protein synthesis (colicins), septum formation (garvicin A), replication and transcription (microcin), or membrane disruption (geobacillin) [51]. They can also facilitate and synergize antibiotic action [52]. Limitations concern their denaturation by digestive proteases, as well as short plasma half-lives and renal toxicity that require extensive chemical engineering (e.g., peptidomimetics and pseudopeptides).

Bacterial predators attack, kill, and enter the periplasm of susceptible Gram-negative bacteria, where they consume the prey cell components [53]. For instance, it was demonstrated that a Bdellovibrio bacteriovorus strain can kill more than 100 human bacterial pathogens, representing a potential alternative or supplement to conventional antibiotics [54].

\section{RNA therapy}

Bacterial sRNAs (the so-called small regulatory RNAs that are $\sim 50$ to 600 nucleotide-long) participate in many regulatory events in bacterial pathogens, including virulence onset [55]. They can also control biofilm formation, antibiotic resistance, and a variety of other bacterial stress responses, some that can reduce the effectiveness of antibiotic treatments 
or be implicated in bacterial persistence, inducing antibiotic tolerance by reduced metabolism [55,56]. Modulating sRNA functions by specific drugs could enhance the efficacy of existing antibiotics, especially if sRNAs impact bacterial growth. There is increasing interest in species-specific drugs for targeting pathogens while leaving the host microbiota unchanged. Since many bacterial sRNAs are only expressed in a few pathogens, targeting those sRNAs would lead to specific antibiotics [57].

Clustered regularly interspaced short palindromic repeat (CRISPR)-based antibacterials are potentially capable to target any bacterial pathogen [58]. Bacterial CRISPR-Cas systems prevent foreign genetic invasions. CRISPR-Cas9 contains an RNA-guided endonuclease inducing RNA targeted DNA breaks with the help of a guide RNA containing complementary base pairs at specific locations within the foreign DNA target. CRISPR derived guide RNAs could be designed to target pathogen-specific virulence or essential genes. Phage-encoded CRISPR-Cas9 can target antibiotic resistance in S. aureus virulent strains [59]. Since CRISPR-Cas targets genomes in a sequence-specific manner, it will distinguish between pathogens and commensals, in turn reducing putative side effects on commensal microbiota. Such technology will allow manipulating complex bacterial populations in a sequence-specific manner that could lead to very specific antibiotics, a step forward for personalized medicine. Weaknesses and difficulties of the RNA therapy are for the therapeutic RNAs to enter the bacterial cells.

\section{Conclusion and perspectives}

Antimicrobial resistance is doubtlessly on the rise. Unchecked, it threatens modern medicine, so there is an urgent need for new antibacterial strategies. As demonstrated here, 
there is a large number of interesting, potentially useful approaches for designing or finding novel antibacterials, but only very few of them have lead so far to their approval as clinically-usable medications. Indeed, the so-called 'crisis of antibiotic crisis' is more a development crisis than a lack of innovation. The main challenges for a successful clinical development of novel antibacterial compounds are primarily financial and have to do with regulatory issues, as reported by pharmaceutical industry professionals [60]. For instance, the most frequent reason for stopping clinical development is due to low return of investment. Therefore, the development of novel antibacterial medicines, especially against MDR bacteria, will imply innovative R\&D, new global regulatory guidance, and innovative trial designs with the help of national and international initiatives, as well as public/private collaborations [60,61]. Because of all these difficulties, it takes 10 to 15 years for a new drug to reach the market from its initial discovery. Around $17 \%$ of antibacterial candidates in pre-clinical development proceed to phase 1 clinical trials and eventually only $\sim 2 \%$ may become approved drugs for clinical use in humans (Figure 2) [46]. The reasons for discontinuing compounds are multiple, such as low activity, high toxicity, unwanted pharmacokinetic profile, resistance development, or various strategic/commercial reasons [46].

As of July 2018, a total of 48 antibiotics (including combinations) and 10 non-conventional (biological) antibacterial agents were in the clinical pipeline that target WHO priority pathogens (mainly ESKAPE), Mycobacterium tuberculosis, and C. difficile (Table 1) [62]. Although there is some progress in the clinical development of new antibacterial drugs, especially against MDR Gram-negative pathogens, the vast majority of them derived from modifications of existing chemical classes [63]. Among the 30 antibiotics that are being 16 
developed for priority pathogens, only 5 fulfill at least one of the four criteria for innovation (i.e., absence of cross-resistance to existing antibiotic classes, new chemical family, now target, or new mechanism of action) [62]. In addition, most of the recently FDA-approved agents also correspond to modified compounds of existing chemical classes (Table 2).

Therefore, really-innovative antibacterial agents under clinical development are scarce, despite numerous approaches involving a wide array of promising 'basic' strategies. The likelihood of marketing and clinical use in humans greatly varies depending on the strategy (Figure 3). The development of naturally-produced and 'me-too' drugs, antibiotic resistance inhibitors, antibiotics with novel mechanisms of action/target, mAbs, and vaccines are more likely to become alternative antibacterials in near future as well as microbiota modifications and phage-derived therapies (since several candidates are currently in the pipeline). More exploratory approaches, requiring substantial developments, are the RNA therapy and immune modulators/virulence suppressors.

Finally, in order to preserve currently-used and future antibacterial agents, we will need to foster effective antibiotic stewardship programs to avoid their overuse/misuse as well as strict infection surveillance, prevention, and control measures to limit the diffusion of MDR bacteria [63].

\section{Acknowledgements}

Kristin R. Baker is thanked for a critical reading of the manuscript. 


\section{Funding}

School of Pharmacy and Medical Sciences of the University of Rennes 1; Agence Nationale pour la Recherche [ANR-15-CE12-0003-01 'sRNA-Fit' to B.F.]; Fondation pour la Recherche Médicale [DBF20160635724 'Bactéries et champignons face aux antibiotiques et antifongiques' to B.F., and personal grant as member of the Scientific Council to V.C.]; Institut National de la Santé et de la Recherche Médicale.

\section{Conflicts of interest}

All authors report no conflicts of interest relevant to this article. 


\section{References}

1. Woolhouse M, Waugh C, Perry MR, Nair H. Global disease burden due to antibiotic resistance - state of the evidence. J Glob Health 2016; 6:010306.

2. Shallcross LJ, Howard SJ, Fowler T, Davies SC. Tackling the threat of antimicrobial resistance: from policy to sustainable action. Philos Trans R Soc Lond B Biol Sci 2015; 370:20140082.

3. Rice LB. Federal funding for the study of antimicrobial resistance in nosocomial pathogens: no ESKAPE. J Infect Dis 2008; 197:1079-81.

4. Theuretzbacher U, Gottwalt S, Beyer P, et al. Analysis of the clinical antibacterial and antituberculosis pipeline. Lancet Infect Dis 2018.

5. Rios AC, Moutinho CG, Pinto FC, et al. Alternatives to overcoming bacterial resistances: state-of-the-art. Microbiol Res 2016; 191:51-80.

6. Charlop-Powers Z, Owen JG, Reddy BV, et al. Global biogeographic sampling of bacterial secondary metabolism. Elife 2015; 4:e05048.

7. Ling LL, Schneider T, Peoples AJ, et al. A new antibiotic kills pathogens without detectable resistance. Nature 2015; 517:455-9.

8. Hover BM, Kim SH, Katz M, et al. Culture-independent discovery of the malacidins as calcium-dependent antibiotics with activity against multidrug-resistant Gram-positive pathogens. Nat Microbiol 2018; 3:41522.

9. Mantravadi PK, Kalesh KA, Dobson RCJ, Hudson AO, Parthasarathy A. The quest for novel antimicrobial compounds: emerging trends in research, development, and technologies. Antibiotics (Basel) 2019; 8:pii: E8.

10. Banik JJ, Brady SF. Recent application of metagenomic approaches toward the discovery of antimicrobials and other bioactive small molecules. Curr Opin Microbiol 2010; 13:603-9.

11. Okada BK, Seyedsayamdost MR. Antibiotic dialogues: induction of silent biosynthetic gene clusters by exogenous small molecules. FEMS Microbiol Rev 2017; 41:19-33.

12. Jampilek J. Design and discovery of new antibacterial agents: advances, perspectives, challenges. Curr Med Chem 2018; 25:4972-5006. 
13. Bush K, Bradford PA. Beta-lactams and beta-lactamase inhibitors: an overview. Cold Spring Harb Perspect Med 2016; 6.

14. Domalaon R, Idowu T, Zhanel GG, Schweizer F. Antibiotic hybrids: the next generation of agents and adjuvants against Gram-negative pathogens? Clin Microbiol Rev 2018; 31.

15. Okano A, Isley NA, Boger DL. Peripheral modifications of [Psi[CH2NH]Tpg(4)]vancomycin with added synergistic mechanisms of action provide durable and potent antibiotics. Proc Natl Acad Sci U S A 2017; 114:E5052-E61.

16. Kwon EJ, Skalak M, Bertucci A, et al. Porous silicon nanoparticle delivery of tandem peptide anti-infectives for the treatment of Pseudomonas aeruginosa lung infections. Adv Mater 2017; 29.

17. Rudramurthy GR, Swamy MK, Sinniah UR, Ghasemzadeh A. Nanoparticles: alternatives against drugresistant pathogenic microbes. Molecules 2016; 21.

18. Karmakar P, Gaitonde V. Promising recent strategies with potential clinical translational value to combat antibacterial resistant surge. Medicines (Basel) 2019; 6:pii: E21.

19. Stratton CW. Phages, fitness, virulence, and synergy: a novel approach for the therapy of infections caused by Pseudomonas aeruginosa. J Infect Dis 2017; 215:668-70.

20. Abedon ST, Garcia P, Mullany P, Aminov R. Phage therapy: past, present and future. Front Microbiol 2017; 8:981.

21. Gordillo Altamirano FL, Barr JJ. Phage therapy in the postantibiotic era. Clin Microbiol Rev. 2019; 32:pii: e00066-18.

22. Fischetti VA. Development of phage lysins as novel therapeutics: a historical perspective. Viruses 2018; 10.

23. Schmelcher M, Donovan DM, Loessner MJ. Bacteriophage endolysins as novel antimicrobials. Future Microbiol 2012; 7:1147-71.

24. Haddad Kashani H, Schmelcher M, Sabzalipoor H, Seyed Hosseini E, Moniri R. Recombinant endolysins as potential therapeutics against antibiotic-resistant Staphylococcus aureus: current status of research and novel delivery strategies. Clin Microbiol Rev 2018; 31.

25. Atkins KE, Flasche S. Vaccination to reduce antimicrobial resistance. Lancet Glob Health 2018; 6:e252. 
26. Lipsitch M, Siber GR. How Can vaccines contribute to solving the antimicrobial resistance problem? MBio 2016; 7.

27. Mutsch M, Zhou W, Rhodes P, et al. Use of the inactivated intranasal influenza vaccine and the risk of Bell's palsy in Switzerland. N Engl J Med 2004; 350:896-903.

28. DiGiandomenico A, Sellman BR. Antibacterial monoclonal antibodies: the next generation? Curr Opin Microbiol 2015; 27:78-85.

29. Kisiela DI, Avagyan H, Friend D, et al. Inhibition and reversal of microbial attachment by an antibody with parasteric activity against the FimH adhesin of uropathogenic E. coli. PLoS Pathog 2015; 11:e1004857.

30. Wilcox MH, Gerding DN, Poxton IR, et al. Bezlotoxumab for prevention of recurrent Clostridium difficile infection. N Engl J Med 2017; 376:305-17.

31. Lehar SM, Pillow T, Xu M, et al. Novel antibody-antibiotic conjugate eliminates intracellular S. aureus. Nature 2015; 527:323-8.

32. Hancock RE, Nijnik A, Philpott DJ. Modulating immunity as a therapy for bacterial infections. Nat Rev Microbiol 2012; 10:243-54.

33. Munguia J, Nizet V. Pharmacological targeting of the host-pathogen interaction: alternatives to classical antibiotics to combat drug-resistant superbugs. Trends Pharmacol Sci 2017; 38:473-88.

34. Hancock RE, Sahl HG. Antimicrobial and host-defense peptides as new anti-infective therapeutic strategies. Nat Biotechnol 2006; 24:1551-7.

35. Seo MD, Won HS, Kim JH, Mishig-Ochir T, Lee BJ. Antimicrobial peptides for therapeutic applications: a review. Molecules 2012; 17:12276-86.

36. Scott MG, Dullaghan E, Mookherjee N, et al. An anti-infective peptide that selectively modulates the innate immune response. Nat Biotechnol 2007; 25:465-72.

37. Roberts PA, Huebinger RM, Keen E, Krachler AM, Jabbari S. Predictive modelling of a novel anti-adhesion therapy to combat bacterial colonisation of burn wounds. PLoS Comput Biol 2018; 14:e1006071.

38. Lynch SV, Wiener-Kronish JP. Novel strategies to combat bacterial virulence. Curr Opin Crit Care 2008; 14:593-9. 
39. Skurnik D, Cywes-Bentley C, Pier GB. The exceptionally broad-based potential of active and passive vaccination targeting the conserved microbial surface polysaccharide PNAG. Expert Rev Vaccines 2016; 15:1041-53.

40. Raffatellu M. Learning from bacterial competition in the host to develop antimicrobials. Nat Med 2018; 24:1097-103.

41. Angelakis E, Merhej V, Raoult D. Related actions of probiotics and antibiotics on gut microbiota and weight modification. Lancet Infect Dis 2013; 13:889-99.

42. van Nood E, Vrieze A, Nieuwdorp M, et al. Duodenal infusion of donor feces for recurrent Clostridium difficile. N Engl J Med 2013; 368:407-15.

43. Wang Y, Venter H, Ma S. Efflux pump inhibitors: a novel approach to combat efflux-mediated drug resistance in bacteria. Curr Drug Targets 2016; 17:702-19.

44. Docquier JD, Mangani S. An update on $\beta$-lactamase inhibitor discovery and development. Drug Resist Updat 2018; 36:13-29.

45. Wong D, van Duin D. Novel beta-lactamase inhibitors: unlocking their potential in therapy. Drugs 2017; $77: 615-28$

46. Farrell LJ, Lo R, Wanford JJ, Jenkins A, Maxwell A, Piddock LJV. Revitalizing the drug pipeline: AntibioticDB, an open access database to aid antibacterial research and development. J Antimicrob Chemother 2018; 73:2284-97.

47. Cabezon E, de la Cruz F, Arechaga I. Conjugation Inhibitors and their potential use to prevent dissemination of antibiotic resistance genes in bacteria. Front Microbiol 2017; 8:2329.

48. Kokai-Kun JF, Roberts T, Coughlin 0 et al. The oral $\beta$-lactamase SYN-004 (ribaxamase) degrades ceftriaxone excreted into the intestine in phase 2a clinical studies. Antimicrob Agents Chemother 2017; 61 :pii: e02197-16.

49. de Gunzburg J, Ducher A, Modess C, et al. Targeted adsorption of molecules in the colon with the novel adsorbent-based medicinal product, DAV132: a proof of concept study in healthy subjects. J Clin Pharmacol 2015; 55:10-6.

50. Izadpanah A, Gallo RL. Antimicrobial peptides. J Am Acad Dermatol 2005; 52:381-90. 
51. Cavera VL, Arthur TD, Kashtanov D, Chikindas ML. Bacteriocins and their position in the next wave of conventional antibiotics. Int J Antimicrob Agents 2015; 46:494-501.

52. Pletzer D, Mansour SC, Hancock REW. Synergy between conventional antibiotics and anti-biofilm peptides in a murine, sub-cutaneous abscess model caused by recalcitrant ESKAPE pathogens. PLoS Pathog 2018; 14:e1007084.

53. Kadouri DE, To K, Shanks RM, Doi Y. Predatory bacteria: a potential ally against multidrug-resistant Gramnegative pathogens. PLoS One 2013; 8:e63397.

54. Mun W, Kwon H, Im H, Choi SY, Monnappa AK, Mitchell RJ. Cyanide Production by Chromobacterium piscinae shields it from Bdellovibrio bacteriovorus HD100 predation. MBio 2017; 8.

55. Waters LS, Storz G. Regulatory RNAs in bacteria. Cell 2009; 136:615-28.

56. Eyraud A, Tattevin P, Chabelskaya S, Felden B. A small RNA controls a protein regulator involved in antibiotic resistance in Staphylococcus aureus. Nucleic Acids Res 2014; 42:4892-905.

57. Colameco S, Elliot MA. Non-coding RNAs as antibiotic targets. Biochem Pharmacol 2017; 133:29-42.

58. Greene AC. CRISPR-based antibacterials: transforming bacterial defense into offense. Trends Biotechnol 2018; 36:127-30.

59. Bikard D, Euler CW, Jiang W, et al. Exploiting CRISPR-Cas nucleases to produce sequence-specific antimicrobials. Nat Biotechnol 2014; 32:1146-50.

60. Bettiol E, Wetherington JD, Schmitt N, Harbarth S; COMBACTE Consortium. Challenges and solutions for clinical development of new antibacterial agents: results of a survey among pharmaceutical industry professionals. Antimicrob Agents Chemother 2015; 59:3695-9.

61. Simpkin VL, Renwick MJ, Kelly R, Mossialos E. Incentivising innovation in antibiotic drug discovery and development: progress, challenges and next steps. J Antibiot (Tokyo) 2017; 70:1087-96.

62. WHO, Update of antibacterial agents in clinical development, July 2018. Available at http://apps.who.int/medicinedocs/documents/s23564en/s23564en.pdf Accessed on 26 February 2019.

63. Talbot GH, Jezek A, Murray BE et al. The infectious diseases society of America's 10 x '20 inititative (ten new systemic antibacterial agents FDA-approved by 2020): is 20 x '20 a possibility? Clin Infect Dis 2019; In press. 


\section{Legends of the figures}

Figure 1. Preventive and therapeutic approaches developed for drug discovery of novel antimicrobial agents. AMP, antimicrobial peptide; MoA, mechanism of action.

Figure 2. Timeline for development phases of novel antibacterial agents. The upper axis represents the average time (in years) of each phase. The percentage of compounds that reaches the stage of clinical development from the previous one is showed. The number of candidate drugs in active clinical development is also indicated for each phase. IND, investigational new drug; MAA (Europe), marketing authorization application; NDA (USA), new drug application. Adapted and data from [46,62].

Figure 3. Likelihood in the near future for alternative strategies currently explored or under development to reach market and clinical use in humans for the treatment or prevention of bacterial infections. S, systemic use; T, topical use. 
Table 1. Antibacterial agents currently in active clinical development (July 2018) [62].

\begin{tabular}{|c|c|c|c|c|}
\hline Name (synonym) & Phase & Antibiotic class & $\begin{array}{c}\text { Route of } \\
\text { administration }\end{array}$ & Innovativeness \\
\hline \multicolumn{5}{|c|}{ Antibiotics and combinations developed for WHO priority pathogens } \\
\hline Eravacycline & $\mathrm{NDA} / \mathrm{MAA}^{a}$ & Tetracycline & iv & - \\
\hline Omadacycline & $\mathrm{NDA}^{a}$ & Tetracycline & iv, oral & - \\
\hline Iclaprim & NDA & DHFR inhibitor & iv & - \\
\hline Lascufloxacin & NDA & Fluoroquinolone & iv, oral & - \\
\hline Imipenem + relebactam & 3 & Carbapenem + DBO-BLI & iv & - \\
\hline Cefiderocol & 3 & Siderophore cephalosporin & iv & \pm \\
\hline Lefamulin & 3 & Pleuromutilin & iv, oral & \pm \\
\hline Sulopenem & 3 & Carbapenem & iv, oral & - \\
\hline Murepavadin & 3 & Membrane-active compound & iv, inhaled & + \\
\hline Aztreonam + avibactam & 3 & Monobactam + DBO-BLI & iv & - \\
\hline Solithromycin & 3 & Macrolide & iv, oral & - \\
\hline Levonadifloxacin/alalevonadifloxacin & 3 & Fluoroquinolone & iv, oral & - \\
\hline Cefilavancin & 3 & Glycopeptide cephalosporin conjugate & iv & \pm \\
\hline Cefepime + AAI-101 & 3 & Cephalosporin + BLI & iv & - \\
\hline Contezolid & $2 / 3$ & Oxazolidinone & iv, oral & - \\
\hline Gepotidacin & 2 & NBTI (triazaacenaphthylene) & iv, oral & + \\
\hline Zoliflodacin & 2 & NBTI (spiropyrimidenetrione) & oral & + \\
\hline ETX2514 + sulbactam & 2 & $\mathrm{DBO}-\mathrm{BLI}+\beta$-lactam-BLI & iv & - \\
\hline Nafithromycin & 2 & Macrolide & oral & - \\
\hline Afabicin & 2 & Fabl inhibitor & iv, oral & + \\
\hline BOS-228 (LYS-228) & 2 & Monobactam & iv & - \\
\hline Cefepime + tazobactam & 2 & Cephalosporin $+\beta$-lactam-BLI & iv & - \\
\hline Ceftibuten + tazobactam (C-Scape) & 2 & Cephalosporin $+\beta$-lactam-BLI & oral & - \\
\hline Colistin + zidovudine & 2 & Polymyxin + nucleoside analogue RT inhibitor & iv & + \\
\hline
\end{tabular}




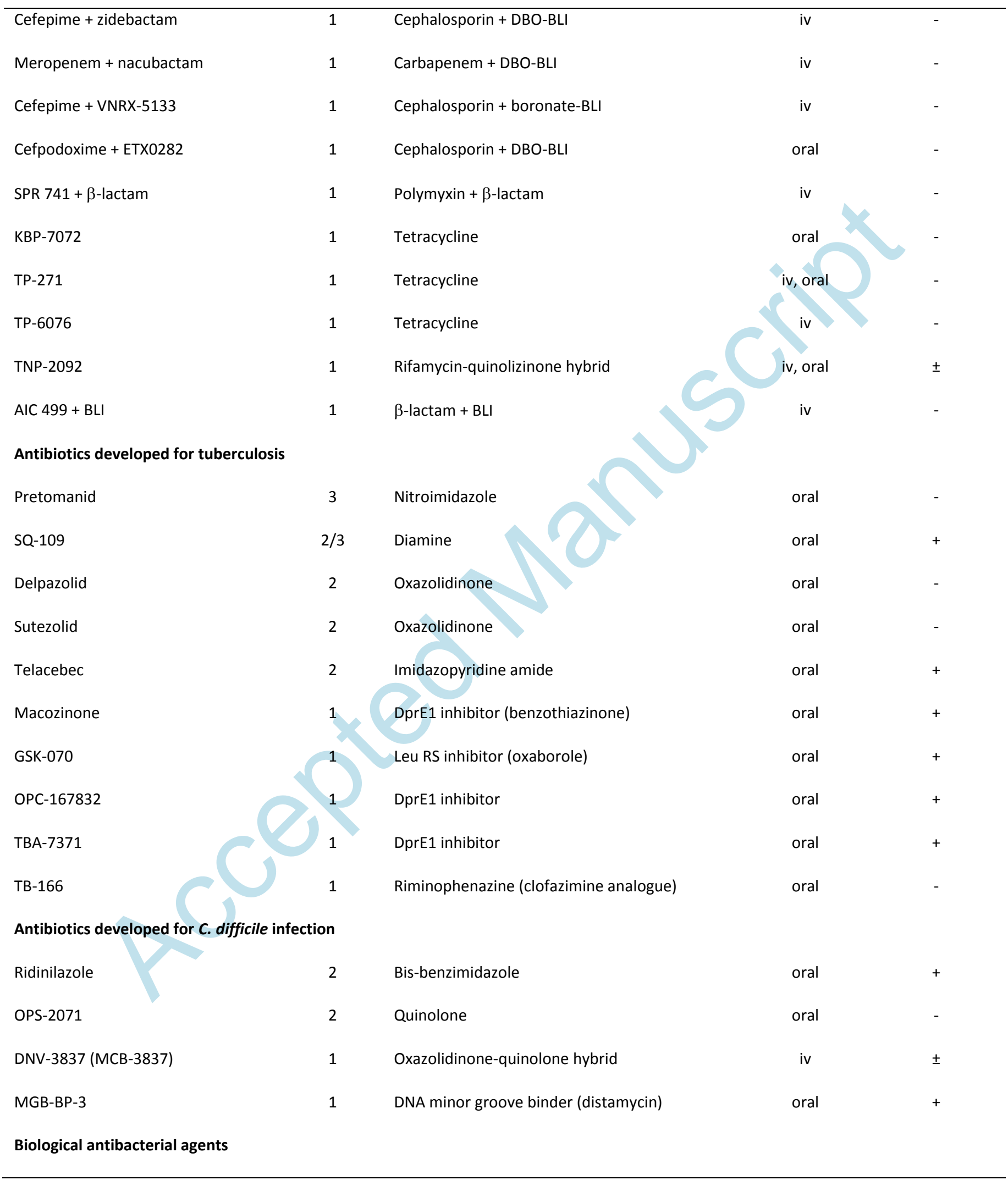




\begin{tabular}{|c|c|c|c|c|}
\hline SAL-200 & 2 & Phage endolysin & iv & n.a. \\
\hline CF-301 & 2 & Phage endolysin & iv & n.a. \\
\hline Suvratoxumab & 2 & Anti-S. aureus IgG monoclonal Ab & iv & n.a. \\
\hline MEDI-3902 & 2 & Anti-P. aeruginosa IgG monoclonal $\mathrm{Ab}$ & iv & n.a. \\
\hline AR-105 & 2 & Anti-P. aeruginosa IgG monoclonal $\mathrm{Ab}$ & iv & n.a. \\
\hline IMM-529 & $1 / 2$ & Anti-C. difficile IgG polyclonal Ab & & n.a. \\
\hline AR-301 (tosatoxumab) & $1 / 2$ & Anti-S. aureus IgM monoclonal Ab & & n.a. \\
\hline $514 G 3$ & $1 / 2$ & Anti-S. aureus IgG monoclonal Ab & & n.a. \\
\hline DSTA 4637S & 1 & Anti-S. aureus IgG monoclonal Ab/rifamycin & iv & n.a. \\
\hline PolyCab & 1 & Anti-C. difficile IgG polyclonal $\mathrm{Ab}$ & iv & n.a. \\
\hline
\end{tabular}

${ }^{a}$ Recently approved by the US FDA (end of 2018).

${ }^{b}$ Innovativeness according to 4 criteria: new chemical class, new target, new mechanism of action and/or absence of cross-resistance to existing antibiotics.

Ab, antibody; BLI, $\beta$-lactam inhibitor; DBO, diazobicyclooctane; DHFR, dihydrofolate reductase; MAA, marketing authorization application; n.a., not applicable; NBTI, novel bacterial topoisomerase II inhibitor; NDA, new drug application; RT, reverse transcriptase. 
Table 2. Antibacterial agents approved by the US FDA from 2009 to 2018 [62].

\begin{tabular}{|c|c|c|c|c|}
\hline Name (synonym) & $\begin{array}{l}\text { Year of } \\
\text { approval }\end{array}$ & Antibiotic class & $\begin{array}{c}\text { Route of } \\
\text { administration }\end{array}$ & Innovativeness $^{\circ}$ \\
\hline \multicolumn{5}{|l|}{ Antibiotics and combinations } \\
\hline Eravacycline & 2018 & Tetracycline & iv & - \\
\hline Omadacycline & 2018 & Tetracycline & iv, oral & - \\
\hline Plazomicin & 2018 & Aminoglycoside & iv & - \\
\hline Meropenem + vabobactam & 2017 & Carbapenem + boronate-BLI & & \pm \\
\hline Delafloxacin & 2017 & Fluoroquinolone & iv, oral & - \\
\hline Ozenoxacin & 2017 & Non-fluorinated quinolone & topical & - \\
\hline Ceftazidime + avibactam & 2015 & Cephalosporin + DBO-BLI & iv & \pm \\
\hline Dalbavancin & 2014 & Lipoglycopeptide & iv & - \\
\hline Oritavancin & 2014 & Lipoglycopeptide & iv & - \\
\hline Tedizolid & 2014 & Oxazolidinone & iv, oral & - \\
\hline Finafloxacin & 2014 & Fluoroquinolone & topical & - \\
\hline Ceftolozane + tazobactam & 2014 & Cephalosporin $+\beta$-lactam-BLI & iv & - \\
\hline Bedaquiline & 2012 & ATP synthase inhibitor & oral & + \\
\hline Fidaxomicin & 2011 & RNA polymerase inhibitor & oral & \pm \\
\hline Ceftaroline & 2010 & Cephalosporin & iv & - \\
\hline Gatifloxacin & 2010 & Fluoroquinolone & topical & - \\
\hline Telavancin & 2009 & Lipoglycopeptide & iv & \pm \\
\hline \multicolumn{5}{|l|}{ Biological antibacterial agents } \\
\hline Bezlotoxumab & 2016 & Anti-C. difficile IgG monoclonal $\mathrm{Ab}$ & iv & n.a. \\
\hline Obiltoxaximab & 2016 & Anti-B. anthracis IgG monoclonal $\mathrm{Ab}$ & inhaled & n.a. \\
\hline Raxibacumab & 2012 & Anti-B. anthracis IgG monoclonal $\mathrm{Ab}$ & inhaled & n.a. \\
\hline
\end{tabular}

Ab, antibody; BLI, $\beta$-lactam inhibitor; DBO, diazobicyclooctane; n.a., not applicable. 


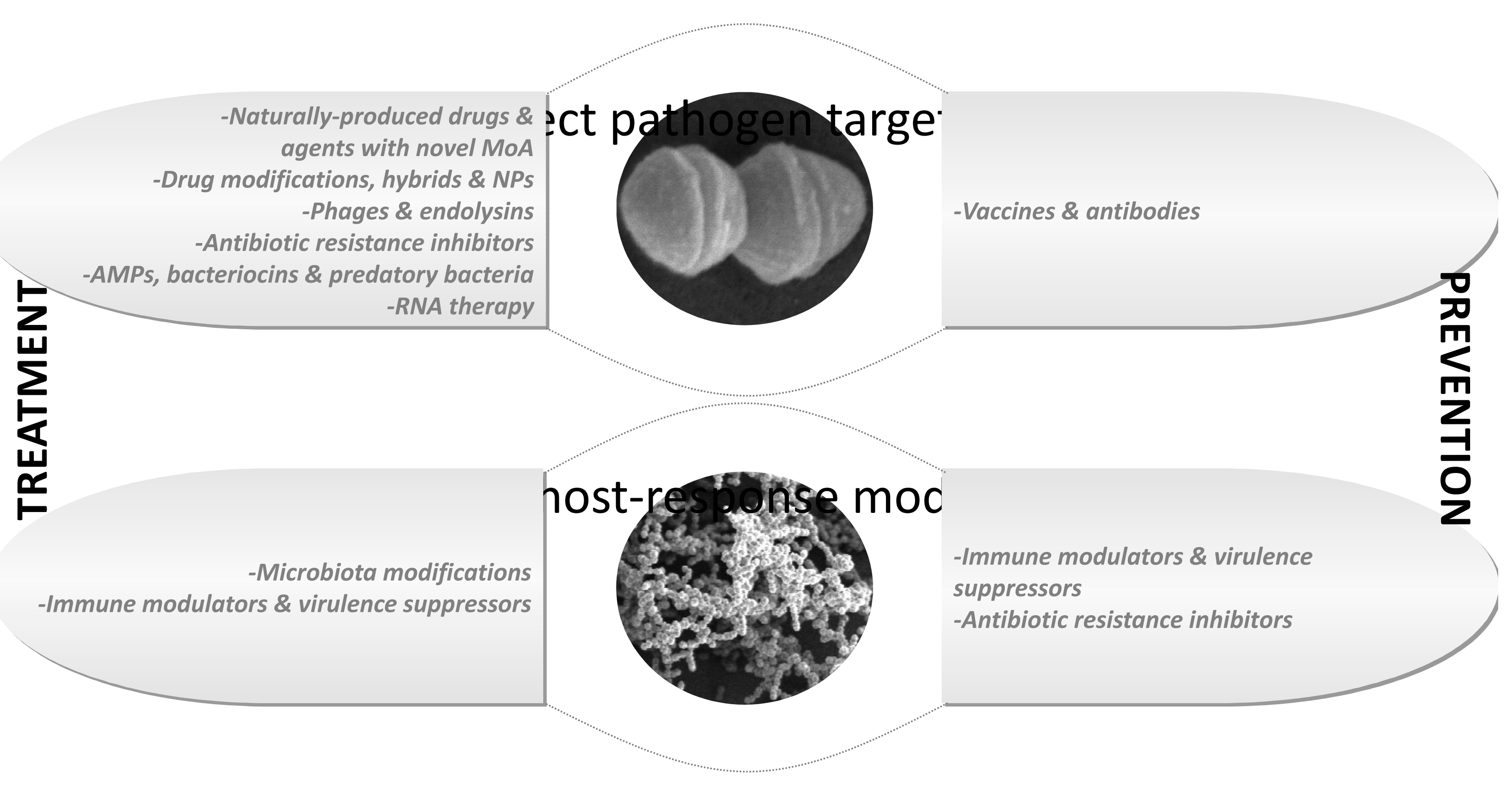




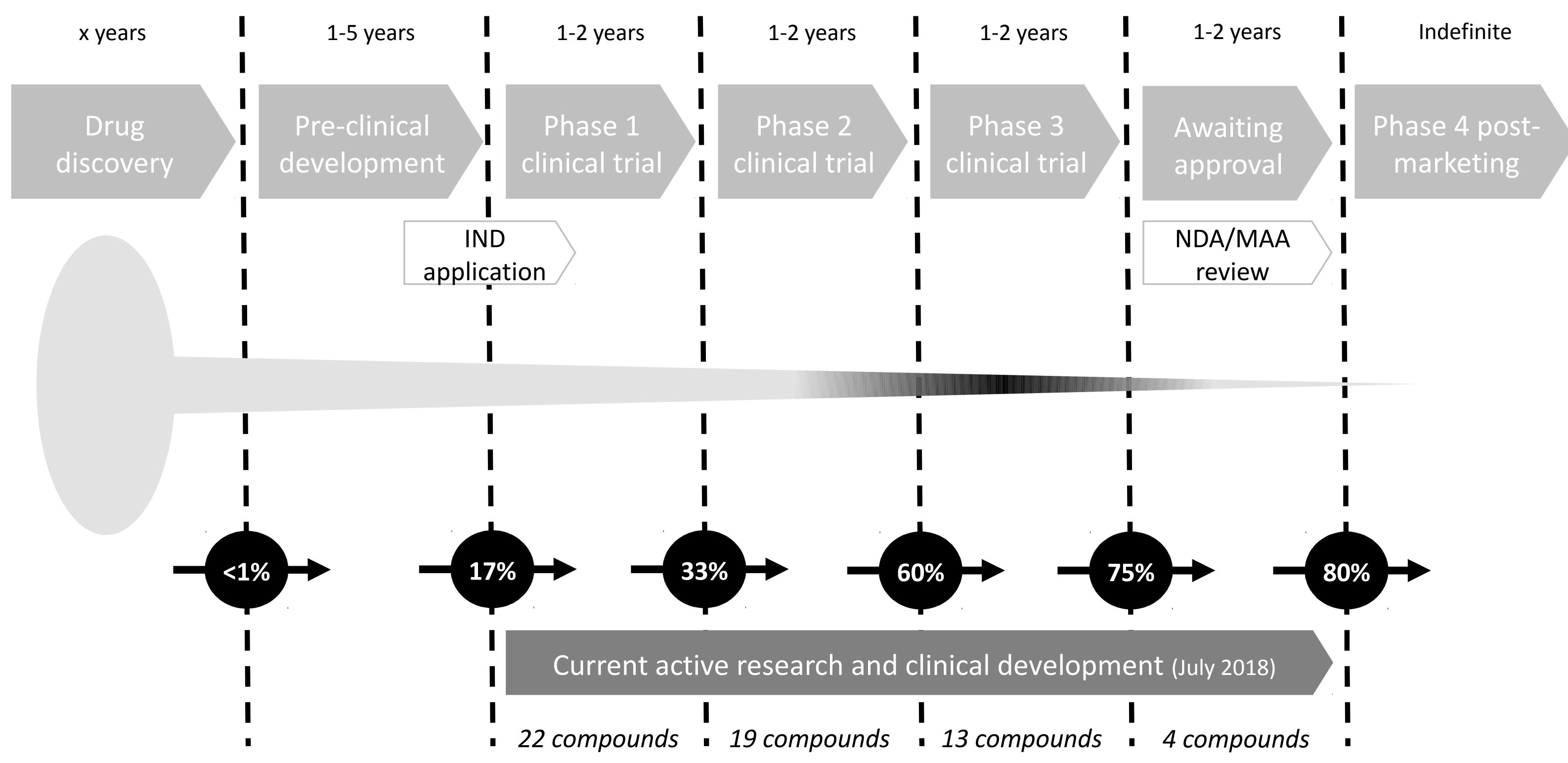




\section{Clinical use}

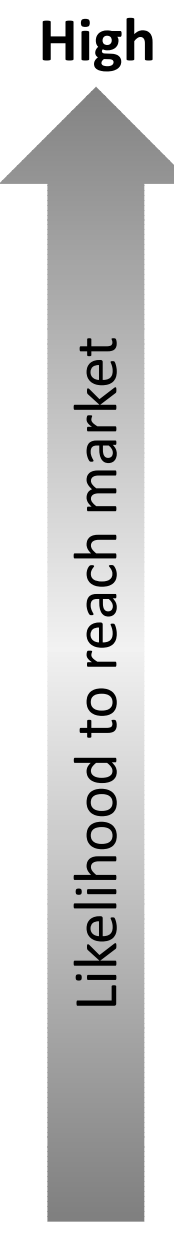

Naturally-produced drugs \& agents with novel MoA $(S, T)$

Drug modifications \& hybrids $(S, T)$

Antibiotic resistance inhibitors (S)

Vaccines \& antibodies (S)

Microbiota modifications (T)

\section{Phages $(T)$ \& endolysins $(S, T)$}

$A M P s$, bacteriocins \& predatory bacteria $(T)$

Immune modulators \& virulence suppressors (S)

Nanoparticules (S)

RNA therapy (S)

Low

\section{Drug discovery}

